\title{
Network analysis revealed aurora kinase dysregulation in five gynecological types of cancer
}

\author{
SHIKHA SUMAN and ASHUTOSH MISHRA \\ Division of Applied Sciences, Indian Institute of Information Technology, Allahabad, Uttar Pradesh 211012, India
}

Received July 8, 2016; Accepted September 13, 2017

DOI: $10.3892 / 01.2017 .7368$

\begin{abstract}
Gene markers are crucial for cancer prognosis and treatment. Previous studies have placed greater emphasis on individual diagnostic genes, thereby ignoring systemic-level attributes across diseases. Female-specific cells namely, breast, endometrium, cervical, ovarian and vulvar cells are highly susceptible to cancer. To date, a limited number of molecular studies have been performed that evaluate common biological processes across gynecological types of cancer. Differentially expressed genes in breast, cervical, endometrial, vulvar and ovarian cancer were utilized to construct protein-protein interaction networks, and to identify a common module across the five cancer types. A single common module with 8 nodes and 26 edges was mined among the five cancer systems. In total, four hub genes were present across the five cancer gene sets. Genes in the common module were enriched for the common pathways and associated diseases. The aurora kinase pathway was revealed to be conserved across the five cancer types surveyed. The present study, therefore, revealed that the aurora kinase pathway has a crucial function in the pathogenesis of the five aforementioned gynecological types of cancer through cross-tumor conservation.
\end{abstract}

\section{Introduction}

Gynecological cancer constitutes $>10 \%$ of cancer cases in the female population globally (1). Major gynecological types of cancer, including cervical, endometrial, ovarian and vulvar cancer, are a cause of concern in females. Breast cancer accounts for $\sim 23 \%$ of all cancer cases among females (2). Diagnosis at advanced stages leads to increased morbidity and mortality, in contrast to an early diagnosis, which increases the chance of a cure and has correspondingly improved survival rates. Diagnostic factors, including age, histology,

Correspondence to: Miss Shikha Suman, Division of Applied Sciences, Indian Institute of Information Technology, Deoghat, Jhalwa, Allahabad, Uttar Pradesh 211012, India

E-mail: rs174@iiita.ac.in

Key words: differentially expressed genes, protein-protein interaction network, module, hubs race, histological grade and stage at diagnosis, along with clinical phenotypes and the degree of causal association of human papillomavirus (HPV) infection (3), and the disease mechanisms of the five aforementioned cancer types appear to be distinct and precise (1). Extensive efforts have been made over recent decades to identify diagnostic genes and develop mechanisms for recognizing cancer in individuals (1). However, a previous study looking to identify prognostic genes has highlighted individual diagnostic genes and their potential function, without scrutinizing the properties and behavior of these diagnostic genes at the systemic level (4).

Studies regarding expression profiles and mutation rates have been conducted to identify common features among various cancer types, although few studies have identified common molecular mechanisms across diverse types of cancer $(1,5,6)$. Certain studies employing novel profiling techniques integrated with existing bioinformatics methods have revealed systemic-level properties emphasizing on networks that instigate the interconnection of genes, proteins and metabolites whose dynamic interactions generate a corresponding function (7) in various types of cancer (5,6,8-11). Previously, one study identified four transcriptional modules associated with the cell cycle and apoptosis in cervical, endometrial and vulvar cancer (1).

System-level attributes may be better understood by utilizing biological networks. Protein-protein interaction (PPI) networks are the most commonly used biological networks for exploiting information on protein interactions. Utilizing expression profiles of the five gynecological types of cancer (cervical, ovarian, vulvar, breast and endometrial), the present study constructed PPI networks for each cancer type, focusing on two main questions regarding these five disease systems. First: Are there genes that are commonly expressed across the five disease systems? Second: Do these commonly expressed genes share similar network properties (including inclusion in hub genes and enrichment in modules) across the five disease systems? The present comparative study revealed common mechanisms and pathways associated with the five gynecological types of cancer.

\section{Materials and methods}

Expression datasets. Gene expression datasets for five gynecological types of cancer, breast, cervical, endometrial, vulvar and ovarian cancer, were retrieved from the Gene Expression Omnibus (https:/www.ncbi.nlm.nih.gov/geo/) (12) for the 
mining of differentially expressed genes (DEGs) in the disease systems for comparison with normal systems. The expression dataset GSE63678 (1) incorporated 5 samples of normal cervical cells and 5 of cervical cancer cells, 5 of normal endometrial cells and 7 of endometrial cancer cells and 7 of vulvar cancer cells and 6 of normal vulvar cells. The dataset GSE57297 (13) included 7 samples of normal and 25 samples of breast cancer cells. The ovarian cancer dataset GSE26712 (14) included 10 samples of normal and 185 samples of ovarian cancer cells.

Analyses of differential expression. Differential expression analyses of the datasets of different cancer types were performed using the Limma (Linear Models for Microarray Data) package (15) in R (16). Pre-processing and normalization were performed to remove noise from the datasets. Adjusted P-values $<0.05$ and Ifold changel $>2$ were taken into account for the identification of DEGs. The P-value was adjusted using the Benjamini-Hochberg method (17) to minimize errors due to multiple hypothesis testing. Overlapping DEGs among the five gynecological types of cancer were selected for further study.

PPI network construction. DEGs of the five cancer types were individually mapped to PPI networks, considering a medium confidence in the Search Tool for the Retrieval of Interacting Genes/Proteins (STRING) version 10.0 (18) database, with Homo sapiens as the source species. Interaction data from high-throughput experiments, genomic contexts, previous knowledge and conserved co-expression are extensively integrated by STRING. The PPI networks were constructed and visualized in Cytoscape (19) version 3.2.

Common module and hubs extraction. The PPI networks for the five gynecological types of cancer were scrutinized for the presence of common sub-network, module and hub genes. A common sub-network was identified by comparing the nodes and edges among the five PPI networks. The common sub-network was further investigated to identify a common high-modularity cluster among the five PPI networks using the Molecular Complex Detection (MCODE) (20) plug-in for Cytoscape. Node score $\geq 0.2$, k-core $=2$, degree $\geq 2$ and maximum depth $=100$ were used as the cut-off criteria for the extraction of common modules. Hub genes were selected from the PPI networks with a connectivity degree of $>25$, and overlapping hubs among the five cancer networks were identified.

Enrichment analyses. Pathway and disease enrichment analyses for the common module genes and common hubs shared between the five gynecological types of cancer were performed using the Web-based Gene Set Analysis Toolkit (21). The cutoff criteria of $\mathrm{P}<0.05$ and number of genes $>2$ were used for enrichment. Hyper-geometric distribution was utilized for enrichment analyses.

\section{Results}

DEGs identification. $\mathrm{P}<0.05$ and Ifold changel $>2$ were used as the demarcating parameters for the identification of
DEGs in the five disease systems. A total of 3,521 DEGs for breast cancer, 1,086 DEGs for cervical cancer, 478 DEGs for endometrial cancer, 2,028 DEGs for ovarian cancer and 728 DEGs for vulvar cancer were identified, all compared with normal samples. The mined DEGs included upregulated and downregulated DEGs. Next, overlapping DEGs among the five cancer disease systems were identified (Fig. 1). A total of 10 DEGs, namely, cyclin B2 (CCNB2), ubiquitin-conjugating enzyme E2C (UBE2C), topoisomerase (DNA) II- $\alpha$ (TOP2A), centromere protein F (CENPF), baculoviral IAP repeat containing 5 (BIRC5), aurora kinase A (AURKA), discs, large (Drosophila) homolog-associated protein 5 (DLGAP5), transforming growth factor- $\beta$ receptor III (TGFBR3), Krüppel-like factor 11 (KLF11) and kinesin family member 20A (KIF20A), were found to be differentially expressed in all five gynecological types of cancer. In total, 29 DEGs were revealed to overlap among cervical, endometrial, breast and ovarian cancer.

PPI network construction. Extracted DEGs from the five cancer datasets were mapped to create PPI networks by utilizing gene-coding protein interaction information. PPI networks for cervical cancer, breast cancer, endometrial cancer, ovarian cancer and vulvar cancer were formed with 689 nodes (4,505 edges), 364 nodes (1,494 edges), 155 nodes (1,005 edges), 733 nodes (5,721 edges) and 542 nodes (1,772 edges), respectively where each node represents a protein and the edge between the nodes represents the interaction between the proteins. These PPI networks were visualized in Cytoscape for further analysis.

Common module and hubs. Overlapping nodes and edges among the five PPI networks were identified to construct a common sub-network among the five gynecological types of cancer. A common module with 8 nodes (MCODE score=7.429; with 26 edges) was extracted from the common sub-network by MCODE. The common modules of the five cancer networks included 8/10 overlapping DEGs: CCNB2, UBE2C, TOP2A, CENPF, BIRC5, AURKA, DLGAP5 and KIF20A (Fig. 2). Additionally, the PPI networks for the five gynecological types of cancer were analyzed for the identification of hub genes exhibiting connectivity degrees of $>25$ (Table I). In total, 4 common hub genes (TOP2A, BIRC5, AURKA and CCNB2) were mined from the five gynecological types of cancer.

Enrichment analyses. Pathway enrichment analyses of the common module genes (Fig. 3A) revealed their association with the cell cycle, mitotic and aurora kinase signaling pathways. Overall, 7 genes from the common module were found to be associated with the cell cycle and mitotic pathways. There were 4 genes, KIF20A, UBE2C, BIRC5 and CENPF, enriched in the mitotic phase-associated pathways. KIF20A, BIRC5, AURKA and DLGAP5 were involved in multiple pathways, including the aurora kinase signaling pathway and the polo-like kinase signaling event in the cell cycle. Moreover, 3/4 common hub genes (TOP2A, BIRC5 and AURKA) were revealed to have a function in the aurora kinase signaling pathway. The genes of the common module were enriched in certain conditions, including cancer, viral infection and adenocarcinoma (Fig. 3B). 
A

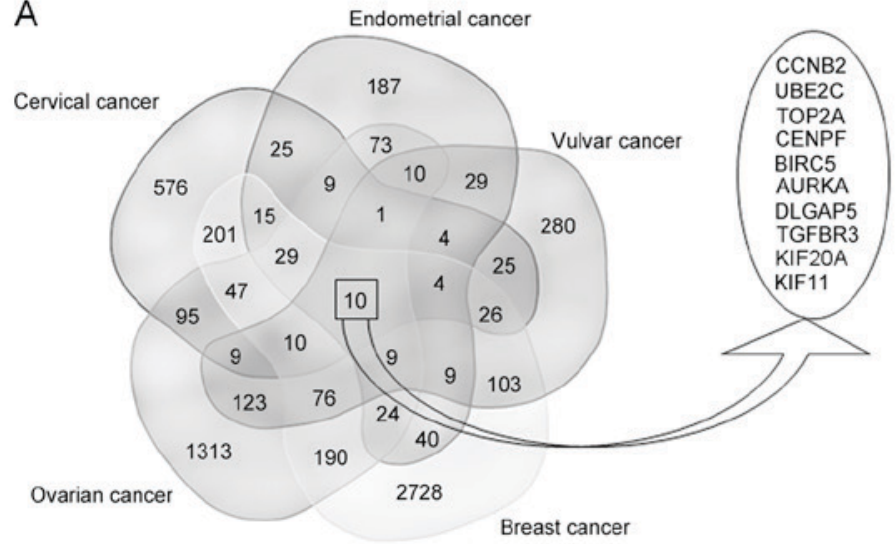

B

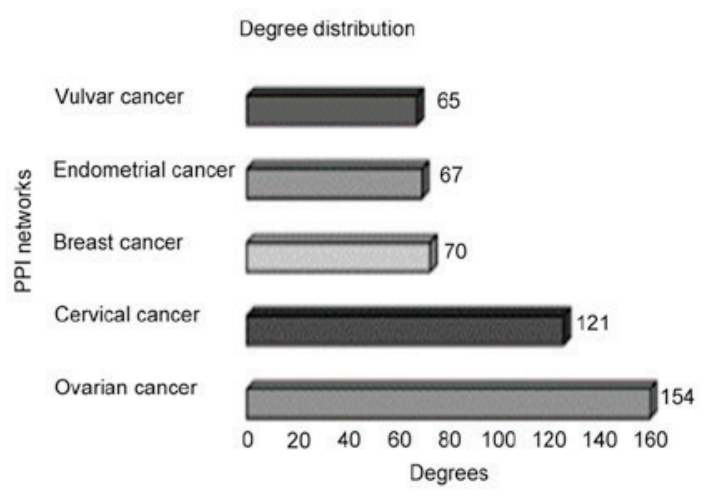

Figure 1. (A) Venn diagram representing the 10 common differently expressed genes among the five female-specific cancer types. (B) Degree distribution of the five cancer PPI networks. Number adjacent to the horizontal represents the maximum degree of the nodes (proteins) in the PPI network. PPI, protein-protein interaction; CCNB2, cyclin 2; UBE2C, ubiquitin-conjugating enzyme E2 C; TOP2A, DNA topoisomerase II- $\alpha$; CENPF, centromere protein F; BIRC5, baculoviral IAP repeat containing 5; AURKA, aurora kinase A; DLGAP5, DLG associated protein 5; TGFBR3, transforming growth factor- $\beta$ receptor 3; KIF20A, kinesin family member 20A; KIF11, kinesin family member 11.

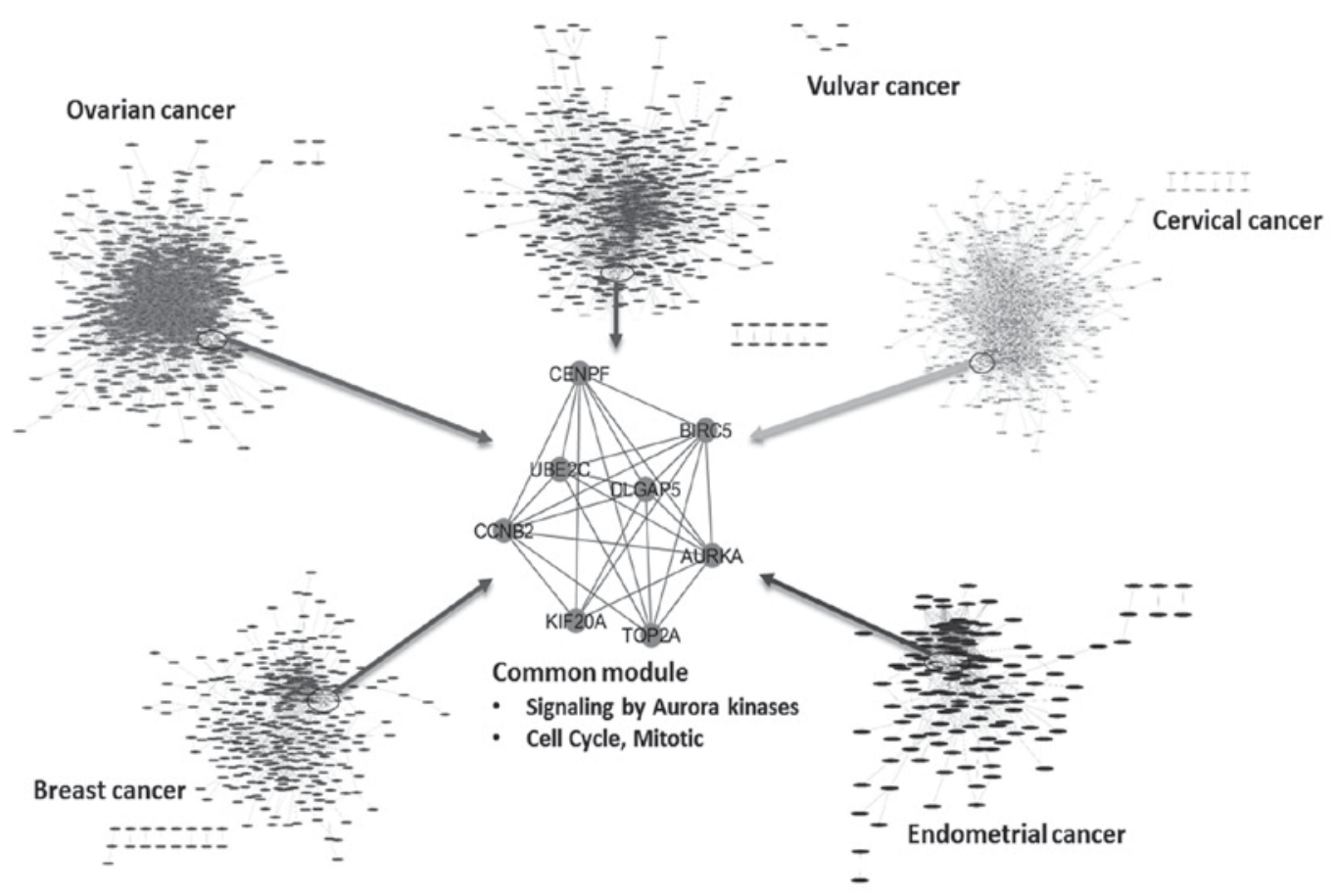

Figure 2. Common modules among the five female-specific cancer types. (A) Pathways enriched; (B) diseases enriched. PLK1, polo-like kinase 1; FOXM1, forkhead box M1.

\section{Discussion}

In the present study, overlapping modules and DEGs among five gynecological types of cancer (breast, cervical, endometrial, vulvar and ovarian cancer) were mined to identify common pathways, which may indicate a common method of tumorigenesis. An integrative approach utilizing differential expression and network analyses was employed for this purpose. It has been hypothesized that cervical, endometrial and vulvar cancer may share common molecular and biochemical features, and the resulting pathways present during cancer development in these three cancer types could shed light on disease development and embryogenesis aspects (1). The present study involved the construction of PPI networks for the five gynecological types of cancer from the gene-encoded protein interaction information, which are differentially expressed at the malignant stage compared with normal tissues. There were 10 DEGs found to be overlapping among the five cancer systems, with 4 DEGs identified as common hubs of the five PPI networks. The PPI networks were investigated to identify overlapping nodes and edges, and to reveal a common sub-network shared by all five female-specific cancer types. There were 4 genes (AURKA, KIF20A, DLGAP5 and BIRC5) involved in aurora kinase signaling pathway activation. Aurora kinase activity peaks during the $\mathrm{G}_{2}$ phase to mitotic phase transition in the cell cycle. KIF20A, UBE2C, BIRC5 
Table I. Hub genes of five female-specific cancer PPI networks.

\section{Cancer type}

Ovarian cancer

Cervical cancer

Endometrial cancer

Breast cancer

Vulvar cancer
Hub genes

AKT1, GAPDH, CDK1, JUN, TOP2A, CCNB1, RAC1, FOS, ACTB, AURKA, HSPA5, ACTA2, BIRC5, CCNB2, CCNA2, CDC20, MMP2, TBP, SMARCA4, ERBB2, MAD2L1, YWHAZ, VEGFA, CDKN2A, TGFB1, BUB1, CDK16, IL8, MCM3, BUB1B, ACTG2, YWHAE, H2AFX, GNB2L1, ISG15, ENO1, TUBA1B, COL1A1, RHOB, TUBA4A, APOE, RACGAP1, MYBL2, TUBB4B, BUB3, RRM2, THBS1, TUBA1C, EIF4G1, PRKDC, HSPB1, MCM7, UBE2C, JUND, COL1A2, TUBB3, NCBP2, SERPINE1, CCNA1, TUBB, KIF2C, PRC1, CCT5, RPL23, TYMS, CALR, HSPD1, CCNE1, PSMB2, PSMB3, TXN, PKM, MYH9, RAD23A, RPL27A, RAD21, SDHC, NEK2, HNRNPC, RPL38, RPL37A, CCT2, HMGB1, FOXM1, OAS1, MMP14, RALA, CALM1, YWHAH, CDCA8, VWF, SDC1, MCL1, ACTN1, PSMC2, SMC4, SNRPE, KIF20A, RBBP4, SNRPF, RPS7, MCM4, KIF4A, SNRPB, OAS3, ACTN4, CFL1, MMP7, CENPF, OASL, ASF1A, RPL36, SMARCB1, BAK1, HSPH1, CTGF, MRPL13, JUNB, COL18A1, SPTAN1, HSPG2, PTBP1, PLK2, RANGAP1, MCM2, ATF3, HSF1, MELK, PPP1CA, SNRPD2, POLR2K, P4HB, COL4A1, SF3A2, ASF1B, PABPN1, DLGAP5, SNCA, CEBPB, PDIA4, PAK2, CLU, PRKCD, COL4A2, ARF1, NUSAP1, POLR2J, RPL36A, SMARCC1, UBA1, RAB8A, COX6A1, POLR2I, TSC2, CEP55, PRPF4

CDK1, TOP2A, PCNA, CDK2, CCNB1, BUB1, MAD2L1, BIRC5, CCNB2, MCM5, AURKA, CDC6, MCM3, CHEK1, MCM4, CENPA, MCM7, KIF11, BUB1B, MCM2, RAD51, NDC80, BRCA1, ESR1, ATR, XPO1, STAT1, RFC4, CDKN2A, KIF2C, NUF2, KIF23, CDC25A, BUB3, PRC1, CENPF, RRM2, RBBP4, MMP9, MCM6, TYMS, SMC4, IL8, RFC3, CCNE1, PIK3CA, KIF20A, FEN1, SMC2, RECQL, KNTC1, TOPBP1, KIF4A, NEK2, MSH2, PRKDC, MCM8, CDC7, CDC25C, WEE1, STAG1, CASC5, RACGAP1, CCNE2, EGR1, ZWILCH, CENPI, MCM10, KIF18A, RFC5, NCAPG, NUP107, ACTA2, MSH6, E2F3, NDE1, PDS5B, DLGAP5, CDC25B, GMPS, WDHD1, NUSAP1, PLK4, FOXM1, ISG15, RBL1, ECT2, CKS2, CENPN, CENPQ, CENPK, TTK, ZWINT, ITGB1, AHCTF1, DSN1, CEP55, ANLN, UBE2C, BARD1, GEN1, DDX58, COL1A1, SGOL2, POLE2, MELK, TRIP13, APOE, DNA2, ASPM, CKS1B, GINS1, EZH2, MMP1, FANCD2, DBF4, ESCO2, PRIM1, YWHAH

CDK1, TOP2A, CCNB1, CDC20, CCNB2, AURKA, BIRC5, MAD2L1, CCNA2, KIF11, BUB1B, PRC1, CENPE, KIF23, KIF4A, KIF2C, CENPA, CDC6, DLGAP5, CENPF, NEK2, ESPL1, KIF20A, RACGAP1, NUSAP1, RRM2, UBE2C, MELK, CEP55, NCAPG

CDK1, TOP2A, PLK1, CCNB2, AURKA, BUB1, BIRC5, CENPA, MMP9, BUB1B, AGT, NDC80, PRC1, CENPE, KIF2C, CDC42, KIF4A, NUF2, CDCA8, ESR1, KIF20A, STAT1, CENPF, DLGAP5, NUSAP1, ANLN

BCL2, VEGFA, NOTCH1, FYN, AR, STAT1, CREB1, PIK3R1, MMP9, KIT, IL8, TOP2A, ACACB, IGF1, VWF, MET, BIRC5, MMP1, FOXO3, GATA3, DCN, YWHAZ, CCNB2, AURKA, IL1B and CENPF were enriched in the mitotic phase-associated pathways, thus assisting in the dysregulation of the aurora kinase pathway. AURKA, TOP2A, KIF20A, CCNB2, UBE2C, BIRC5 and CENPF were enriched in the dysregulation of the cell cycle and mitotic pathways, which in turn could affect the normal function of the aurora kinase signaling pathways. In total, 7/8 genes from the common module were involved in the dysregulation of the aurora kinase signaling pathway.

The aurora kinase family is comprised of 3 serine-threonine protein kinases: Aurora kinase A, aurora kinase B and aurora kinase $\mathrm{C}$, and the aurora kinase $\mathrm{A}$ protein is encoded by the AURKA gene. This family of proteins are regulators of cell division (22). Since the aurora kinase pathway is involved in chromatin duplication, mitotic entry, centromere maturation, chromatin condensation, spindle assembly and bipolar spindle formation (Fig. 4A) (23), overexpression or dysregulation of the associated pathways may lead to an increased rate of cell division and then to tumorigenesis.
Overexpression of aurora kinase has been reported in breast, liver, pancreatic, bladder, thyroid and gastrointestinal cancer types (24). AURKA overexpression has been proposed to be associated with aneuploidy in breast cancer (25). Polymorphisms in the AURKA gene have been shown to increase the risk of primary breast cancer (26). Studies have revealed that the increased expression of AURKA is associated with laryngeal, nasopharyngeal and breast cancer metastasis (27-30). AURKA has been demonstrated to be accountable for the phosphorylation of Breast Cancer 1, Early Onset (BRCA1) (31). Tanaka et al (32) investigated the immunohistochemical analysis of invasive ductal adenocarcinomas of the breast, that revealed the overexpression of aurora kinases in a majority of cases. Miyoshi et al (33) investigated the correlation of aurora kinase A mRNA expression with numerous clinic pathological factors and CIN in breast cancer. Nadler et al (34) investigated the correlation of a population of patients with a decreased survival rate based on aurora kinase A expression, 
A

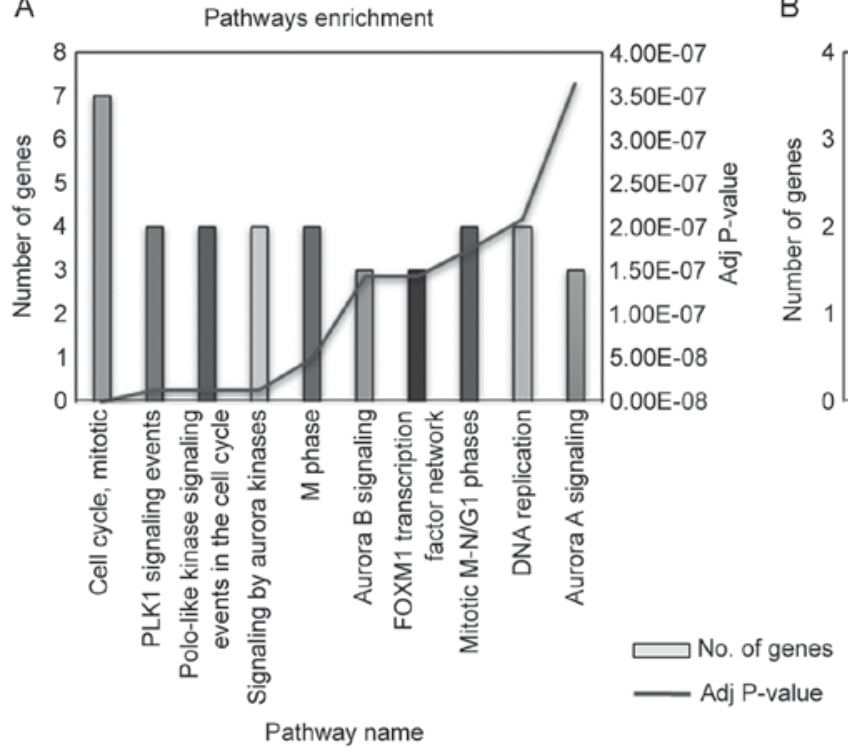

B

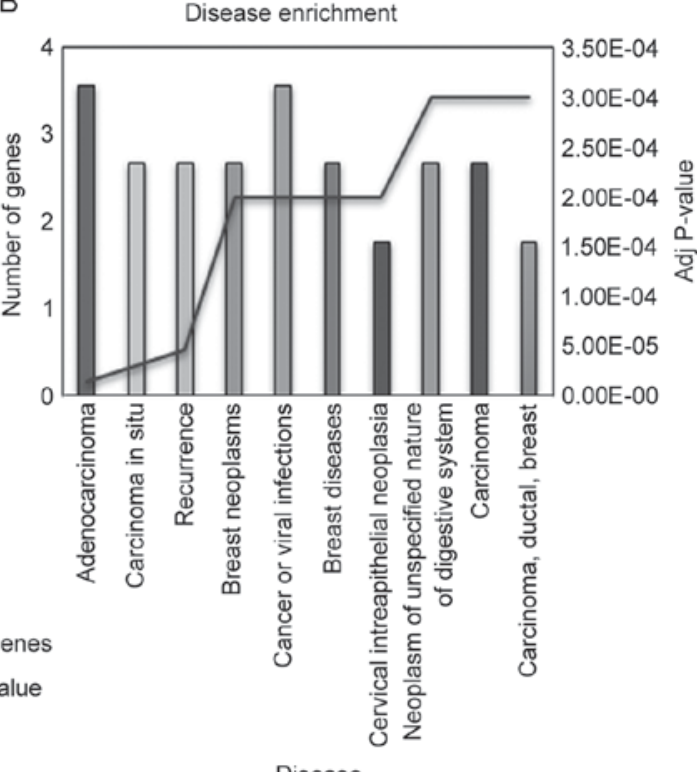

Figure 3. (A) Common pathway enrichment and (B) disease enrichment analyses of the common module genes by considering the number of genes and adjusted P-values. MAD2, mitotic arrest deficient 2 like 1; MCAK, kinesin family member 2C; NF- $\mathrm{BB}$, nuclear factor $\kappa$-light-chain-enhancer of activated B cells; RalA, RAS like proto-oncogene A; BRCA1, breast cancer type 1 susceptibility protein; BUBR1, mitotic checkpoint serine/threonine-protein kinase BUB1- $\beta$; AURKA, aurora kinase A.

A

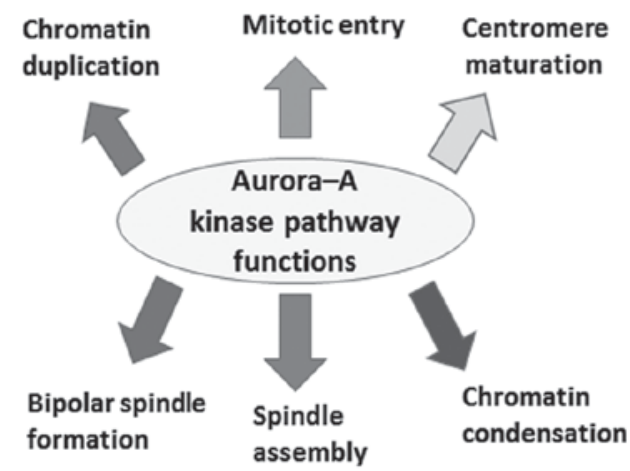

B

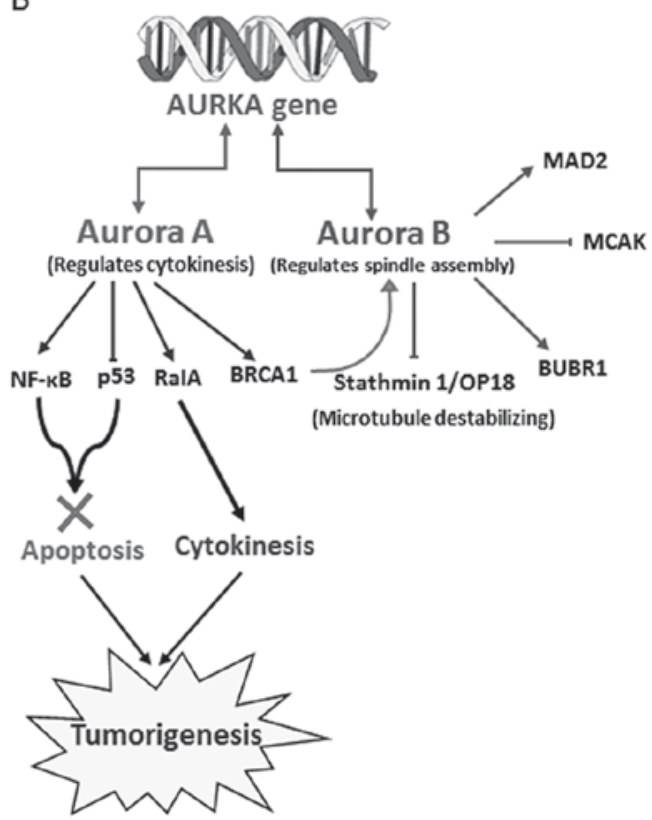

Figure 4. (A) Aurora kinase A and aurora kinase B interact with oncogenes to initiate tumorigenesis. (B) Function of the AURKA pathway. CENPF, centromere protein F; BIRC5, baculoviral IAP repeat containing 5; UBE2C, ubiquitin-conjugating enzyme E2 C; DLGAP5, DLG associated protein 5; CCNB2, cyclin 2; AURKA, aurora kinase A; KIF20A, kinesin family member 20A; TOP2A, DNA topoisomerase II- $\alpha$.

whereas aurora kinase B does not interfere with the survival of patients. Thus, aurora kinase A was proposed to be a preferred drug target in breast cancer. Studies have indicated that aurora kinase A and aurora kinase B are significantly overexpressed in carcinoma and in cervical intraepithelial neoplasia 3 (CIN3), compared with in the normal cervix (35). A previous study found that the overexpression of aurora kinase A is crucial for survival of HPV-transformed cervical cancer cells (36).
Overexpression of aurora kinase A is observed in $83 \%$ of human epithelial ovarian carcinoma cases (37). It has been proposed that AURKA regulates cell migration and adhesion in epithelial ovarian cancer (38). Human chromosome 20q13.2, which contains AURKA, is usually amplified in ovarian cancer (39). Furthermore, Aurora kinase A has been revealed to be associated with Federation Internationale de Gynecologie et d'Obstetrique stage, tumor grade and survival (37). AURKA and AURKB 
are overexpressed in endometrial cancer, in comparison with normal proliferative tissues, as determined by Kurai et al (40). Moreno-Bueno et al (41) revealed the presence of high AURKA expression levels in type-II adenocarcinoma via the microarray analysis of endometrial cancer tissue. Few studies are available that highlight the potential mechanisms for vulvar cancer (42). It has been revealed to originate from two distinct pathways: HPV-dependent and HPV-independent (43).

KIF20A belongs to the kinesin superfamily, which is involved in essential cellular functions including migration, mitosis and intracellular transport though interaction with microtubules (44). KIF20A has been proposed to be a potential immunotherapeutic target for cancer (45) and is often overexpressed in breast cancer, lung cancer, pancreatic cancer, gastric cancer, bladder cancer, melanoma and other malignancies. (46-48). KIF20A small interfering RNA (siRNA), along with six other siRNAs, has been reported to kill HeLa cervical cancer cells (49). Another study revealed KIF20A to be a hub of the gene co-expression network of endometrial cancer (50).

BIRC5 is a member of the inhibitor of apoptosis family, which is involved in mitosis and protection against apoptotic cell death (51). Signaling networks required for tumor maintenance are expected to be compromised by disabling BIRC5 and survivin, thereby enhancing its utility as a cancer drug target (52). Genomic copy number variations in BIRC5 have been proposed to be involved in the progression of breast cancer (53). Furthermore, BIRC5 expression may result in breast tumor proliferation by promoting genetic instability (54). BIRC5 has been reported to be overexpressed in cervical cancer (55). Overexpression of BIRC5 promotes cervical cancer progression and metastasis (55). BIRC5 has been reported to be overexpressed in various human malignancies, including ovarian cancer (56) and endometrial cancer (57). Human telomerase reverse transcriptase is an enzyme that enables cells to divide indefinitely and overcome replicative senescence (58); this enzyme has been reported to be upregulated in vulvar intraepithelial neoplasia, with the emergence of resistance to apoptosis and cellular longevity via survivin activation (58).

Silencing of DLGAP suppresses tumorigenicity and inhibits cellular proliferation by inducing cell cycle arrest at the $\mathrm{G}_{2} / \mathrm{M}$ phase (59). DLGAP5 has been proposed to be a target of the neurogenic locus notch homolog protein 3 signaling pathway (59), which is associated with embryogenesis and breast cancer tumorigenesis (60). DLAGP5 is reportedly a member of the cervical cancer interaction network (61), and has been revealed to be overexpressed in ovarian carcinoma compared with in normal ovarian epithelium (59). DLGAP5 is overexpressed in endometrial cancer tissues, in comparison with normal endometrial tissues (62).

Overexpression of aurora kinases not only dysregulates the cell cycle but also facilitates interactions with several cancer-associated proteins, including tumor protein p53, nuclear factor $\kappa$-light-chain enhancer of activated $\mathrm{B}$ cells $(\mathrm{NF}-\kappa \mathrm{B}), \mathrm{v}-\mathrm{Myc}$, Ras and pathways including the mitogen-activated protein kinase/extracellular-related kinase and phosphoinositide-3 kinase/protein kinase B signaling pathways (Fig. 4B) $(63,64)$. In the present study, the overexpression of these oncogenes was also observed. Aurora kinase $\mathrm{A}$ is essential for cytokinesis as it activates $\mathrm{NF}-\kappa \mathrm{B}$, an anti-apoptotic gene that is involved in growth regulation of cells, the overexpression of which leads to cancer (65). Additionally, p53 induces apoptosis; the overexpression of aurora kinase A phosphorylates p53 and inhibits its function. (22). Aurora kinase A may phosphorylate RalA, thus influencing Ras signaling and leading to tumorigenesis (66). Myc was also overexpressed in the present analysis. Myc is a quintessential oncogene, which is overexpressed in cancer cells and functions as an angiogenic switch (67). Myc has been revealed to be present in number of cancer types, including colorectal, prostate and breast cancer (68). Aurora kinase B overexpression leads to chromosomal segregation error and abnormal cytokinesis, potentially causing carcinogenesis (63).

Aurora kinase A has been revealed to have a vital function in increasing chemotherapy resistance, cell exhibiting stem-cell-like properties and mesenchymal phenotypes (69). Thus, the inhibition of aurora kinase A has demonstrated an increased sensitivity and decreased proliferation of breast cancer to chemotherapy and hormonal treatments (70). The aurora kinase B inhibitor ZM447439 has already been proven to suppress the growth of cervical cancer cells, thus enhancing chemosensitivity (71). A previous study has confirmed the potential utility of the aurora kinase A inhibitor alisertib, when used in combination with taxanes, as a therapeutic strategy for ovarian cancer (72). To verify the clinical significance of AURKA in improving the therapy of endometrial cancer, a combination treatment utilizing AURKA inhibitors and paclitaxel is proposed (73).

DEGs identified from the microarray analysis of the five gynecological types of cancerous cell sample were utilized for the construction of PPI networks in the present study. Identification and analysis of the common hub genes and modules suggested the presence of the aurora kinase pathway in all five of the gynecological types of cancer. Aurora kinases are a potentially promising area of research, as they are overexpressed in five gynecological types of cancer and are key regulators of cell division, as well as acting together with a number of oncogenes. The findings of the present study may be utilized for the development of exhaustive models of carcinogenesis, which may lead to the development of novel therapeutic approaches that target mechanisms frequently altered across various cancer types. However, further experimental validations are required to confirm these findings.

\section{Acknowledgements}

Computing facilities at the Indian Institute of Information Technology (Allahabad, India) are acknowledged.

\section{References}

1. Pappa KI, Polyzos A, Jacob-Hirsch J, Amariglio N, Vlachos GD, Loutradis D and Anagnou NP: Profiling of discrete gynecological cancers reveals novel transcriptional modules and common features shared by other cancer types and embryonic stem cells. PLoS One 10: e0142229, 2015.

2. Mohankumar K, Pajaniradje S, Sridharan S, Singh VK, Ronsard L, Banerjea AC, Benson CS, Coumar MS and Rajagopalan R: Mechanism of apoptotic induction in human breast cancer cell, MCF-7, by an analog of curcumin in comparison with curcumin-an in vitro and in silico approach. Chem Biol Interact 210: 51-63, 2014. 
3. US Cancer Statistics Working Group: United States Cancer Statistics: 1999-2011 incidence and mortality web-based report. U.S. Department of Health and Human Services, Center for Disease Control and Prevention and National Cancer Institute Atlanta, GA, 2014. Available: www.cdc.gov/uscs. Accessed 3 March 2015

4. Yang Y,Han L, Yuan Y, Li J, Hei N and Liang H: Gene co-expression network analysis reveals common system-level properties of prognostic genes across cancer types. Nat Commun 5: 3231, 2014.

5. Peng L, Bian XW, Li DK, Xu C, Wang GM, Xia QY and Xiong Q: Large-scale RNA-seq transcriptome analysis of 4043 cancers and 548 normal tissue controls across 12 TCGA cancer types. Sci Rep 5: 13413, 2015

6. Martinez E, Yoshihara K, Kim H, Mills GM, Treviño V and Verhaak RG: Comparison of gene expression patterns across 12 tumor types identifies a cancer supercluster characterized by TP53 mutations and cell cycle defects. Oncogene 34: 2732-2740, 2015.

7. Alberghina L, Höfer T and Vanoni M: Molecular networks and system-level properties. J Biotechnol 144: 224-33, 2009.

8. Alisoltani A, Fallahi H, Ebrahimi M, Ebrahimi M and Ebrahimie E: Prediction of potential cancer-risk regions based on transcriptome data: towards a comprehensive view. PLoS One 9: e96320, 2014.

9. Mine KL, Shulzhenko N, Yambartsev A, Rochman M, Sanson GF, Lando M, Varma S, Skinner J, Volfovsky N, Deng T, et al: Gene network reconstruction reveals cell cycle and antiviral genes as major drivers of cervical cancer. Nat Commun 4: 1806, 2013

10. Hoadley KA, Yau C, Wolf DM, Cherniack AD, Tamborero D, Ng S, Leiserson MDM, Niu B, McLellan MD, Uzunangelov V, et al: Multiplatform analysis of 12 cancer types reveals molecular classification within and across tissues of origin. Cell 158: 929-944, 2014.

11. Fruzangohar M, Ebrahimie E, Ogunniyi AD, Mahdi LK, Paton JC and Adelson DL: Correction: Comparative GO: A web application for comparative gene ontology and gene ontology-based gene selection in bacteria. PLoS One 10: e0125537, 2015.

12. Barrett T, Wilhite SE, Ledoux P, Evangelista C, Kim IF, Tomashevsky M, Marshall KA, Phillippy KH, Sherman PM, Holko M, et al: NCBI GEO: Archive for functional genomics data sets-update. Nucleic Acids Res 41: D991-D995, 2013.

13. Fu J, Allen W, Xia A, Ma Z and Qi X: Identification of biomarkers in breast cancer by gene expression profiling using human tissues. Genom Data 2: 299-301, 2014.

14. Bonome T,Levine DA, Shih J, Randonovich M, Pise-Masison CA, Bogomolniy F, Ozbun L, Brady J, Barrett JC, Boyd J and Birrer MJ: A gene signature predicting for survival in suboptimally debulked patients with ovarian cancer. Cancer Res 68 5478-5486, 2008.

15. Gautier L, Cope L, Bolstad BM and Irizarry RA: Affy-analysis of affymetrix genechip data at the probe level. Bioinformatics 20 : 307-315, 2004

16. Team RCore: R: A language and environment for statistical computing. Vienna, Austria: R Foundation for Statistical Computing: 3-36, 2014. http://www.r-project.org/.

17. Benjamini $\mathrm{Y}$ and Hochberg Y: Controlling the false discovery rate: A practical and powerful approach to multiple testing. Journal of the Royal Statistical Society. J R Statics Soc B: 289-300, 1995.

18. Von Mering C, Huynen M, Jaeggi D, Schmidt S, Bork P and Snel B: STRING: A database of predicted functional associations between proteins. Nucleic Acids Res 31: 258-261, 2003.

19. Smoot ME, Ono K, Ruscheinski J, Wang PL and Ideker T: Cytoscape 2.8: New features for data integration and network visualization. Bioinformatics 27: 431-432, 2011.

20. Bader GD and Hogue CW: An automated method for finding molecular complexes in large protein interaction networks. BMC Bioinformatics 4: 2, 2003

21. Wang J, Duncan D, Shi Z and Zhang B: Web-based gene set analysis toolkit (WebGestalt): Update 2013. Nucleic Acids Res 41: W77-W83, 2013.

22. Dar AA, Goff LW, Majid S, Berlin J and El-Rifai W: Aurora kinase inhibitors-rising stars in cancer therapeutics? Mol Cancer Ther 9: 268-278, 2010.

23. Siegel RL, Miller KD and Jemal A: Cancer statistics, 2015. CA Cancer J Clin 65: 5-29, 2015.

24. Carvajal RD, Tse A and Schwartz GK: Aurora kinases: New targets for cancer therapy. Clin Cancer Res 12: 6869-75, 2006.
25. Xia LP, Zhou FF, Yang MT and Liu Q: Roles of Aurora-A in tumorigenesis and prognosis of breast cancer. Ai Zheng 28: 668-672, 2009 (In Chinese).

26. Cox DG, Hankinson SE and Hunter DJ: Polymorphisms of the AURKA (STK15/Aurora Kinase) gene and breast cancer risk (United States). Cancer Causes Control 17: 81-83, 2006

27. Yang LY, He CY, Chen XH, Su LP, Liu BY and Zhang H: Aurora kinase A revives dormant laryngeal squamous cell carcinoma cells via FAK/PI3K/Akt pathway activation. Oncotarget 7: 48346-48359, 2016.

28. Lo Iacono ML, Monica V, Saviozzi S, Ceppi P, Bracco E, Papotti $M$ and Scagliotti GV: Aurora Kinase A expression is associated with lung cancer histological-subtypes and with tumor de-differentiation. J Transl Med 9: 100, 2011.

29. Staff S, Isola J, Jumppanen M and Tanner M: Aurora-A gene is frequently amplified in basal-like breast cancer. Oncol Rep 23: 307-312, 2010.

30. Zou Z, Yuan Z, Zhang Q, Long Z, Chen J, Tang Z, Zhu Y, Chen S, Xu J, Yan M, et al: Aurora kinase A inhibition-induced autophagy triggers drug resistance in breast cancer cells. Autophagy 8: 1798-1810, 2012.

31. Ouchi M, Fujiuchi N, Sasai K, Katayama H, Minamishima YA, Ongusaha PP, Deng C, Sen S, Lee SW and Ouchi T: BRCA1 phosphorylation by Aurora-A in the regulation of G2 to M transition. J Biol Chem 279: 19643-19648, 2004.

32. Tanaka T, Kimura M, Matsunaga K, Fukada D, Mori H and Okano Y: Centrosomal kinase AIK1 is overexpressed in invasive ductal carcinoma of the breast. Cancer Res 59: 2041-2044, 1999.

33. Miyoshi Y, Iwao K, Egawa C and Noguchi S: Association of centrosomal kinase STK15/BTAK mRNA expression with chromosomal instability in human breast cancers. Int J Cancer 92: 370-373, 2001.

34. Nadler Y, Camp RL, Schwartz C, Rimm DL, Kluger HM and Kluger Y: Expression of Aurora A (but not Aurora B) is predictive of survival in breast cancer. Clin Cancer Res 14: 4455-4462, 2008.

35. Twu NF, Yuan CC, Yen MS, Lai CR, Chao KC, Wang PH, $\mathrm{Wu} \mathrm{HH}$ and Chen YJ: Expression of Aurora kinase A and B in normal and malignant cervical tissue: High Aurora A kinase expression in squamous cervical cancer. Eur J Obstet Gynecol Reprod Biol 142: 57-63, 2009.

36. Gabrielli B, Bokhari F, Ranall MV, Oo ZY, Stevenson AJ, Wang W, Murrell M, Shaikh M, Fallaha S, Clarke D, et al: Aurora A is critical for survival in HPV-transformed cervical cancer. Mol Cancer Ther 14: 2753-2761, 2015.

37. Campos SM and Ghosh S: A current review of targeted therapeutics for ovarian cancer. J Oncology 2010: 149362, 2010.

38. Do TV, Xiao F, Bickel LE, Klein-Szanto AJ, Pathak HB, Hua X, Howe C, O'Brien SW, Maglaty M, Ecsedy JA, et al: Aurora kinase A mediates epithelial ovarian cancer cell migration and adhesion. Oncogene 33: 539-549, 2014.

39. Katsha A, Belkhiri A, Goff L and El-Rifai W: Aurora kinase A in gastrointestinal cancers: Time to target. Mol Cancer 14: 106, 2015.

40. Kurai M, Shiozawa T, Shih HC, Miyamoto T, Feng YZ, Kashima H, Suzuki A and Konishi I: Expression of Aurora kinases A and B in normal, hyperplastic and malignant human endometrium: Aurora B as a predictor for poor prognosis in endometrial carcinoma. Hum Pathol 36: 1281-1288, 2005.

41. Moreno-Bueno G, Sánchez-Estévez C, Cassia R, Rodríguez-Perales S, Díaz-Uriarte R, Domínguez O, Hardisson D, Andujar M, Prat J, Matias-Guiu X, et al: Differential gene expression profile in endometrioid and nonendometrioid endometrial carcinoma STK15 is frequently overexpressed and amplified in nonendometrioid carcinomas. Cancer Res 63: 5697-5702, 2003.

42. Van de Nieuwenhof HP, van der Avoort IA and de Hullu JA Review of squamous premalignant vulvar lesions. Crit Rev Oncol Hematol 68: 131-156, 2008

43. Ueda Y, Enomoto T, Kimura T, Yoshino K, Fujita M and Kimura T: Two distinct pathways to development of squamous cell carcinoma of the vulva. J Skin Cancer 2011: 951250, 2011

44. Miki H, Setou M, Kaneshiro K and Hirokawa N: All kinesin superfamily protein, KIF, genes in mouse and human. Proc Natl Acad Sci USA 98: 7004-7011, 2001.

45. Tomita Y, Yuno A, Tsukamoto H, Senju S, Kuroda Y, Hirayama M, Irie A, Kawahara K, Yatsuda J, Hamada A, et al: Identification of promiscuous KIF20A long peptides bearing both CD4+ and CD8+ T-cell epitopes: KIF20A-specific CD4+ T-cell immunity in patients with malignant tumor. Clin Cancer Res 19: 4508-4520, 2013 
46. Imai K, Hirata S, Irie A, Senju S, Ikuta Y, Yokomine K, Harao M, Inoue M, Tomita Y, Tsunoda T, et al: Identification of HLA-A2-restricted CTL epitopes of a novel tumour-associated antigen, KIF20A, overexpressed in pancreatic cancer. Br J Cancer 104: 300-307, 2011.

47. Yamashita J, Fukushima S, Jinnin M, Honda N, Makino K, Sakai K, Masuguchi S, Inoue Y and Ihn H: Kinesin family member 20A is a novel melanoma-associated antigen. Acta Derm Venereol 92: 593-597, 2012.

48. Claerhout S, Lim JY, Choi W, Park YY, Kim K, Kim SB, Lee JS, Mills GB and Cho JY: Gene expression signature analysis identifies vorinostat as a candidate therapy for gastric cancer. PLoS One 6: e24662, 2011.

49. Groth-Pedersen L, Aits S, Corcelle-Termeau E, Petersen NH, Nylandsted J and Jäättelä M: Identification of cytoskeleton-associated proteins essential for lysosomal stability and survival of human cancer cells. PloS one 7: e45381, 2012.

50. Chou WC, Cheng AL, Brotto $M$ and Chuang CY: Visual gene-network analysis reveals the cancer gene co-expression in human endometrial cancer. BMC genomics 15: 300, 2014.

51. Marivin A, Berthelet J, Plenchette S and Dubrez L: The inhibitor of apoptosis (IAPs) in adaptive response to cellular stress. Cells 1: 711-737, 2012.

52. Sawyers C: Targeted cancer therapy. Nature 432: 294-297, 2004.

53. Ghaffari K, Hashemi M, Ebrahimi E and Shirkoohi R: birc5 genomic copy number variation in early-onset breast cancer. Iran Biomed J 20: 241-245, 2016.

54. Boidot R, Vegran F, Jacob D, Chevrier S, Gangneux N, Taboureau J, Oudin C, Rainville V, Mercier L and Lizard-Nacol S: The expression of BIRC5 is correlated with loss of specific chromosomal regions in breast carcinomas. Genes, Chromosomes Cancer 47: 299-308, 2008.

55. Zhou XL and Wang M: Expression levels of survivin, Bcl-2 and KAI1 proteins in cervical cancer and their correlation with metastasis. Genet Mol Res 14: 17059-17067, 2015.

56. Ferrandina G, Legge F, Martinelli E, Ranelletti FO, Zannoni GF, Lauriola L, Gessi M, Gallotta V and Scambia G: Survivin expression in ovarian cancer and its correlation with clinico-pathological, surgical and apoptosis-related parameters. Br J Cancer 92: 271-277, 2005.

57. Zahedi P, Aminimoghaddam S, Sayahpour FA, Haghpanah V, Amiri P, Fereidoni F, Mahrampour E, Larijani B, Tavakkoly-Bazzaz J and Amoli MM: Association of survivin gene polymorphism with endometrial cancer. Int J Gynecol Cancer 22: 35-37, 2012.

58. Wellenhofer A and Brustmann $\mathrm{H}$ : Expression of human telomerase reverse transcriptase in vulvar intraepithelial neoplasia and squamous cell carcinoma: An immunohistochemical study with survivin and p53. Arch Pathol Lab Med 136: 1359-1365, 2012.

59. Chen X, Thiaville MM, Chen L, Stoeck A, Xuan J, Gao M, Shih IeM and Wang TL: Defining NOTCH3 target genes in ovarian cancer. Cancer Res 72: 2294-2303, 2012.
60. Smalley M, Piggott L and Clarkson R: Breast cancer stem cells: Obstacles to therapy. Cancer Lett 338: 57-62, 2013.

61. Luo Y, Wu Y, Peng Y, Liu X, Bie J and Li S: Systematic analysis to identify a key role of CDK1 in mediating gene interaction networks in cervical cancer development. Ir J Med Sci 185: 231-239, 2016.

62. Risinger JI, Allard J, Chandran U, Day R, Chandramouli GV, Miller C, Zahn C, Oliver J, Litzi T, Marcus C, et al: Gene expression analysis of early stage endometrial cancers reveals unique transcripts associated with grade and histology but not depth of invasion. Front Oncol 3: 139, 2013.

63. Katayama $\mathrm{H}$ and Sen S: Aurora kinase inhibitors as anticancer molecules. Biochim Biophy Acta 1799: 829-839, 2010.

64. Lai CH, Tseng JT, Lee YC, Chen YJ, Lee JC, Lin BW, Huang TC, Liu YW, Leu TH, Liu YW, et al: Translational up-regulation of Aurora-A in EGFR-overexpressed cancer. J Cell Mol Med 14: 1520-1531, 2010

65. Sun C, Chan F, Briassouli P and Linardopoulos S: Aurora kinase inhibition downregulates NF- $\mathrm{B}$ and sensitises tumour cells to chemotherapeutic agents. Biochem Biophys Res Commun 352: 220-225, 2007.

66. Lim KH, Brady DC, Kashatus DF, Ancrile BB, Der CJ, Cox AD and Counter CM: Aurora-A phosphorylates, activates and relocalizes the small GTPase RalA. Mol Cell Biol 30: 508-523, 2010.

67. Nilsson JA and Cleveland JL: Myc pathways provoking cell suicide and cancer. Oncogene 22: 9007-9021, 2003.

68. Cole MD: MYC association with cancer risk and a new model of MYC-mediated repression. Cold Spring Harb Perspect Med 4: a014316. 2014.

69. D'Assoro AB, Liu T, Quatraro C, Amato A, Opyrchal M, Leontovich A, Ikeda Y, Ohmine S, Lingle W, Suman V, et al: The mitotic kinase Aurora-a promotes distant metastases by inducing epithelial-to-mesenchymal transition in $\mathrm{ER} \alpha(+)$ breast cancer cells. Oncogene 33: 599-610, 2014

70. Opyrchal M, Sangameswaran KD, Khoury T, Boland P, Galanis E and Haddad TC: Aurora Kinase inhibitors in breast cancer treatment. American Journal of Hematology/Oncology 11, 2015.

71. Zhang L and Zhang S: ZM447439, the Aurora kinase B inhibitor, suppresses the growth of cervical cancer SiHa cells and enhances the chemosensitivity to cisplatin. J Obstet Gynaecol Res 37: 591-600, 2011.

72. Do TV, Xiao F, Bickel LE, Klein-Szanto AJ, Pathak HB, Hua X, Howe C, O'Brien SW, Maglaty M, Ecsedy JA, et al: Aurora kinase A mediates epithelial ovarian cancer cell migration and adhesion. Oncogene 33: 539-549, 2014.

73. Umene K, Yanokura M, Banno K, Irie H, Adachi M, Iida M, Nakamura K, Nogami Y, Masuda K, Kobayashi Y, et al: Aurora kinase $\mathrm{A}$ has a significant role as a therapeutic target and clinical biomarker in endometrial cancer. Int J Oncol 46: 1498-1506, 2015. 